In the Review by Brett A English, Kelan Thomas, Jack Johnstone, Adam Bazih, Lev Gertsik and Larry Ereshefsky, 'Use of translational pharmacodynamic biomarkers in early-phase clinical studies for schizophrenia', which appeared in the January 2014 issue of Biomarkers in Medicine (8[1], 29-49 [2014]; http://www.futuremedicine.com/doi/pdf/10.2217/bmm.13.135), it has been brought to our attention that Figure 1 was incorrectly published as:

(A)

\title{
SAD (HNVs)
}

MAD (HNVs or patients)

$$
\text { Dose levels up to }
$$

$1 \mathrm{a}$

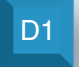

$1 \mathrm{c}$

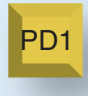

$2 a / b$

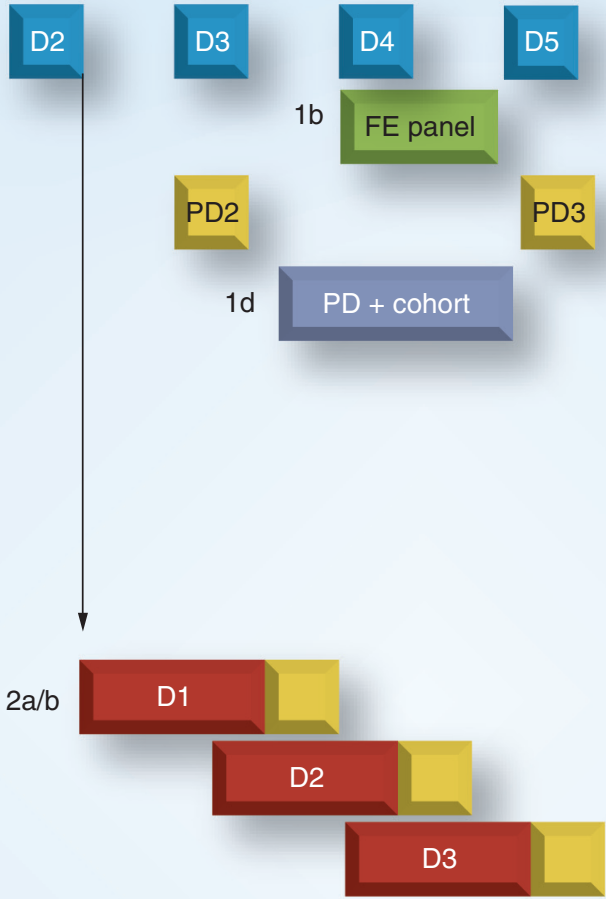

$2 \mathrm{C}$

PD + cohort 1a (SAD MTD): traditional SAD study conducted in HNVs or jump to patients depending on AEs to determine MTD

1b (FE): conducted at cohort 4 using dose 3 upon clearance of PK from cohort 3 (dose 3)

1c (SAD-PD): 2-3 dose levels (PD1-3); CSF (PK + PD), qEEG, fMRI or PET in HNVs, patients (may defer to MAD)

1d (PD+): pharmacologic reversal study including qEEG and fMRI occurring upon safety/tolerability data from prior cohorts

2a (SAD MTD): traditional SAD study conducted in HNVs or patients to determine MTD

2b (PD): PD methods incorporated into $M A D$, which may include qEEG, imaging and cognitive ratings. Dosing may extend beyond Css

2c (PD+): shorter (ex. 7 day) study combining BSL and last dose assessments

(B)

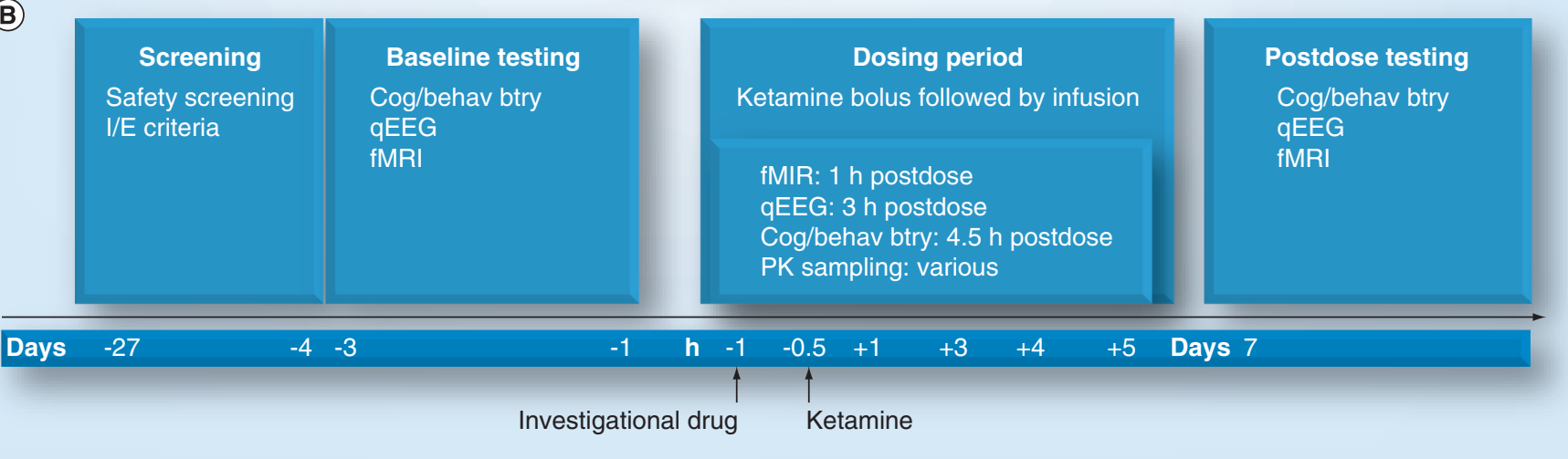

The correct Figure $\mathbf{1}$ is shown overleaf: 
(A)

SAD (HNVs)

Dose levels up to

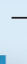

D1

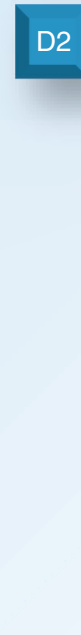

MAD (HNVs or patients)
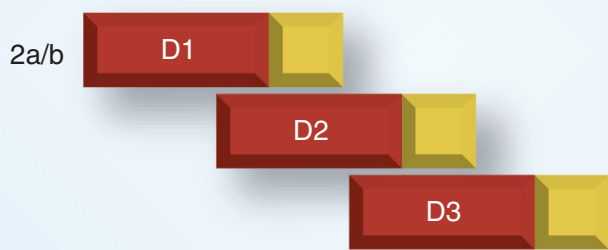

2c

\section{PD + cohort}

1a (SAD MTD): traditional SAD study conducted in HNVs or jump to patients depending on AEs to determine MTD

1b (FE): conducted at cohort 4 using dose 3 upon clearance of PK from cohort 3 (dose 3 )

1c (SAD-PD): 2-3 dose levels (PD1-3); CSF (PK + PD), qEEG, fMRI or PET in HNVs, patients (may defer to MAD)

1d (PD+): pharmacologic reversal study including $\mathrm{qEEG}$ and $\mathrm{fMRI}$ occurring upon safety/tolerability data from prior cohorts

2a (SAD MTD): traditional SAD study conducted in HNVs or patients to determine MTD

2b (PD): PD methods incorporated into $M A D$, which may include $q E E G$, imaging and cognitive ratings. Dosing may extend beyond Css

2c (PD+): shorter (ex. 7 day) study combining BSL and last dose assessments

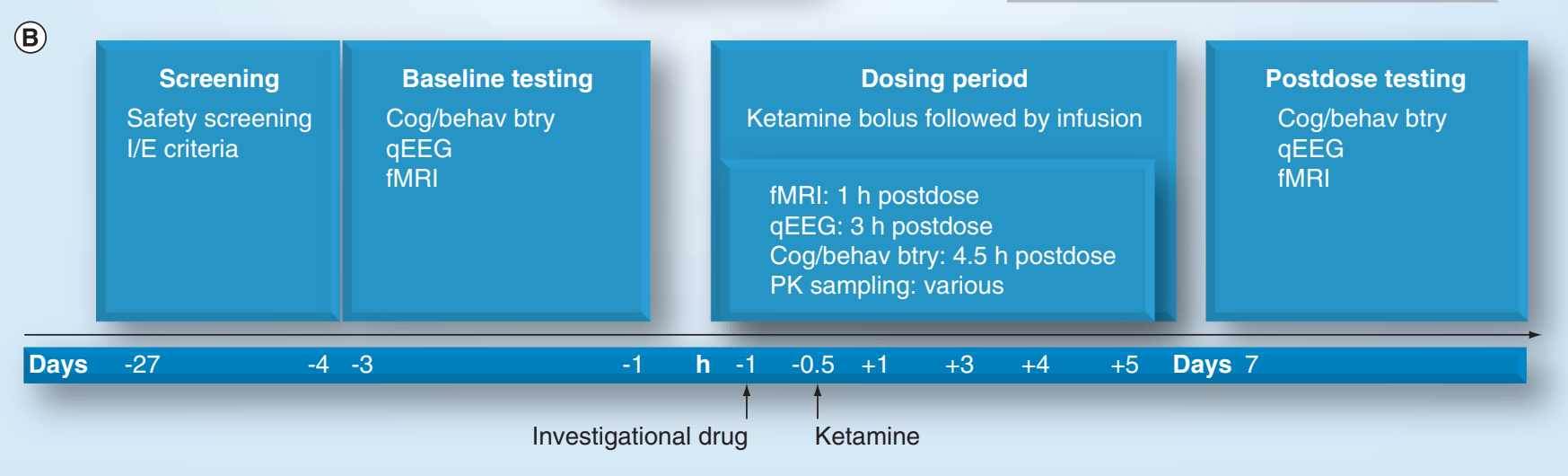

The editors of Biomarkers in Medicine would like to sincerely apologize for any confusion or inconvenience this may have caused. 